\title{
Opioid Crisis
}

Article 1: Opioid-Related Factors Affecting Mortality in Indiana, USA

Sariya Udayachalerm, PhD Candidate; David R. Foster, PharmD, FCCP; Jane Wang, PhD;

Michael D. Murray, PharmD, MPH, FCCP

Article 2: Opioid Overdose Mortalities: Are There Racial Disparities?

Marwa Rawy, PharmD; Kevin Look, PhD; Betty Chewning, PhD

Article 3: Consequences of 2014 Legislation on Controlled Substance Dispensing Patterns and Utilization of the Indiana Prescription Drug Monitoring Program: A Three-Year Kristin R. Villa, PharmD, MS; Kimberly S. Plake, PhD, FAPhA; Alan J. Zillich, PharmD, FCCP Matthew M. Murawski, PhD

Article 4: Design of a Qualtrics Online Survey to Assess Prescription Drug Monitoring Program Use Using a Factorial Survey Design

Brahmendra Reddy Viyyuri, PharmD; Matthew J Witry, PharmD, PhD;

Barbara St. Marie, PhD, ANP-BC, GNP-BC

Article 5: Using the Theory of Planned Behavior to Explore Opioid Disposal Behaviors Arveen Kaur, PharmD, MPH; David A Mott, PhD, FAPhA, RPh

Article 6: Establishing Inter- Reliability for a Coding Protocol for Analyzing Video Recordings for Opioid Consults Between Pharmacy Students and Standardized Patients Tanvee Thakur, BPharm, MS; Betty Chewning, PhD 


\section{Opioid-Related Factors Affecting Mortality in Indiana, USA}

Sariya Udayachalerm, PhD Candidate

PhD Candidate

Purdue University

College of Pharmacy

640 Eskenazi Avenue

Indianapolis, IN 46202

sudayach@purdue.edu

David R. Foster, PharmD, FCCP

Associate Professor

Purdue University

College of Pharmacy

640 Eskenazi Avenue

Indianapolis, IN 46202

drfoster@purdue.edu

\author{
Jane Wang, PhD \\ Senior Data Analyst \\ Regenstrief Institute \\ $1101 \mathrm{~W} .10^{\text {th }}$ Street \\ Indianapolis, IN 46202 \\ janwang@regentrief.org
}

\author{
Michael D. Murray, PharmD, MPH, FCCP, FISPE \\ Distinguished Professor of Pharmacy Practice \\ Purdue University College of Pharmacy \& \\ Endowed Chair of Medication Safety Senior Scientist, \\ Regenstrief Institute \\ $1101 \mathrm{~W} .10^{\text {th }}$ Street \\ Indianapolis, IN 46202 \\ mdmurray@regenstrief.org
}

Acknowledgements: The data of this study was managed by Regenstrief Institute, Indiana University School of Medicine

\section{ABSTRACT}

Background: Opioid abuse and overdose in the U.S. is a public health crisis largely fueled by widespread use of prescription opioids for chronic nonmalignant pain.

Objective: To determine factors affecting mortality of patients in the Indiana Network for Patient Care (INPC).

Methods: INPC is a 30-year health information exchange (HIE) data captured from Indiana's health systems including 17.2 million patients. This study used patient, pharmacy, and mortality data between 01/01/2012-12/31/2017.

Subjects were $\geq 18$ years old with first opioid prescriptions (index) within study period without opioid in the past 12 months. Exclusion criteria included cancer, dementia, and/or liver diseases. Outcomes included death and number of days from index to death.

Survival analyses and Kaplan-Meier plots were used to determine survival function and graph time to death. Cox-proportional hazards model used death as the dependent variable controlling for sex, age, race, number of opioid prescriptions, day supply, dose (morphine milligram equivalents; MME), short/long acting opioids, and concurrent benzodiazepine. SAS 9.4 was used for analysis and $p<0.05$ was considered statistically significant.

Results: Total 1,812,239 opioid prescriptions were identified for 385,025 subjects. Mean age was 53.1 years ( \pm 18.0 ) with $58.2 \%$ female and $83.5 \%$ white race. Mean number of opioid prescriptions was 2 prescriptions/person (range: 1, 188), median daily MME was $5 \mathrm{mg}$ (range: $0.02,240$ ), and median day supply was 5 days/prescription/person (range: 1, 120).

Hydrocodone was mostly prescribed (59.0\%). At the end of study period, 3410 patients $(0.89 \%)$ died with the median of days from index to death of $682(1,2182)$. Compared to other race groups, risk of death was higher for African American patients $(1.31$ [95\%Cl: $1.09,1.57, \mathrm{p}<0.0001])$. Other significant factors included higher age $(1.083$ [95\% Cl: 1.080, 1.086], $\mathrm{p}<0.0001)$, male sex $(1.54$ [95\%Cl: $1.44,1.65], p<0.0001)$, number of opioid prescriptions (0.994 [95\% $\mathrm{Cl}: 0.991,0.998], p=0.0012)$, average MME dose $(1.017$ [95\%Cl: $1.014,1.019], p<0.0001)$, day supply $(1.020$ [95\% Cl: $1.016,1.024], p<0.0001)$, using concurrent short and long acting opioids (1.88 [95\%Cl: 1.67, 2.11], $\mathrm{p}<0.0001)$, and concurrent benzodiazepine $(1.38[95 \% \mathrm{Cl}: 1.27,1.50], \mathrm{p}<0.0001)$.

Conclusion: Using a statewide HIE data, we found that race, age, sex, number of opioid prescriptions, MME, day supply, concurrent short and long acting opioids, and concurrent benzodiazepine are associated with mortality.

Key Words: Opioid, Mortality, Indiana 


\section{Opioid Overdose Mortalities: Are There Racial Disparities?}

Marwa Rawy, Pharm.D.

Graduate Student

University of Wisconsin-Madison

School of Pharmacy

777 Highland Ave

Madison, WI 53705

rawy@wisc.edu

Kevin Look, Ph.D.

Assistant Professor

University of Wisconsin-Madison

School of Pharmacy

777 Highland Ave

Madison, WI 53705

kevin.look@wisc.edu
Betty Chewning, Ph.D.

Professor

University of Wisconsin-Madison

School of Pharmacy

777 Highland Ave

Madison, WI 53705

betty.chewning@wisc.edu

\section{ABSTRACT}

Objectives: With increases in deaths due to prescription and illicit opioids, a growing concern among white populations, the opioid crisis has been described as a "White epidemic" given that blacks are less likely to be prescribed opioid analgesics especially for nontraumatic/nonsurgical pain compared to their white counterparts. This study aims to compare death rates between blacks/African Americans and whites with respect to (1) All opioid-related deaths, and (2) Deaths due to commonly prescribed opioids, synthetic opioids (mostly illicit fentanyl), and heroin.

Methods: The latest available 2016 mortality data were extracted from the National Vital Statistics System (NVSS) using the CDC WONDER query tool. Age-adjusted death rates involving any opioid, prescription opioids, synthetic opioids, and heroin were identified using relevant ICD-10 codes at the national and state levels for both blacks and whites. Rate ratios were calculated to compare ageadjusted deaths rates.

Results: In 2016, there were 42,249 opioid-related deaths with whites and blacks accounting for $87.5 \%$ and $10.7 \%$ respectively of the decedents. Nationally, whites were $34 \%$ more likely to die due to any opioid overdose compared to blacks. This percentage, however, increased to $45 \%$ for prescription opioids and declined to $21 \%$ for synthetic opioids, and $20 \%$ for heroin. There were variabilities in opioid-related death rates at the state level. In some states, especially in the Midwest, blacks were disproportionately more likely to die from an opioid overdose than whites. These disparities were higher for illicit opioids.

Conclusions: The wide gap in death rates from prescription opioids among whites compared to blacks is diminishing for illicit opioids. With the sharp recent rise in overdose fatalities from illicit compared to prescription opioids and with the disparities in death rates black populations are experiencing in some states, it is important that policymakers include blacks/African Americans in their conversation about the opioid epidemic.

Key Words: Disparities, Race, Opioid Mortality, Prescription Opioids, Illicit Opioids 


\section{Consequences of 2014 Legislation on Controlled Substance Dispensing Patterns and Utilization of the Indiana Prescription Drug Monitoring Program: A Three-Year Review}

Kristin R. Villa, PharmD, MS

Graduate Student

Department of Pharmacy Practice

College of Pharmacy

Purdue University

575 Stadium Mall Dr.

West Lafayette, IN 47906

kvilla@purdue.edu

Kimberly S. Plake, PhD, FAPhA

Assistant Dean

Purdue University

College of Pharmacy

575 Stadium Mall Dr.

West Lafayette, IN 47906

kplake@purdue.edu

\author{
Alan J. Zillich, PharmD, FCCP \\ William S Bucke Professor and Head \\ Department of Pharmacy Practice \\ Purdue University \\ College of Pharmacy \\ 575 Stadium Mall Dr. \\ West Lafayette, IN 47906 \\ azillich@purdue.edu \\ Matthew M. Murawski, PhD \\ Associate Professor \\ Department of Pharmacy Practice \\ Purdue University \\ College of Pharmacy \\ 575 Stadium Mall Dr. \\ West Lafayette, IN 47906 \\ murawski@purdue.edu
}

Acknowledgements: This study was partially funded by a Purdue Research Foundation (PRF) Gran

\section{ABSTRACT}

Introduction: The misuse of prescription controlled substances is a growing issue in the United States. Specifically, the use and abuse of opioids has been recognized as a public health emergency, but other categories of controlled substances are also misused. In addition to opioids, the therapeutic classes of medications associated with prescription drug misuse include benzodiazepines, stimulants, and sedative-hypnotics. According to the 2016 National Survey on Drug Use and Health (NSDUH), approximately 6.2 million Americans misused prescription drugs within the last month and 18.7 million within the last year, which is the second highest reported incidence of misuse for any category after marijuana. New laws and regulations have been put in place both at federal and state levels to address the misuse of these prescription medications.

Objectives: 1. To describe trends in the controlled substances dispensed in Indiana during a three-year period. 2. To determine the effect of federal and state-specific legislative and regulatory changes relating to opioids and prescription drug monitoring programs on controlled substance dispensing in Indiana and use of Indiana's prescription drug monitoring program, INSPECT.

Methods: Retrospective analysis of all de-identified Indiana prescription controlled substance dispensations and practitioner queries within the INSPECT system from January 1, 2014 through December 31, 2016.

Preliminary Results: Over 38 million individual dispensations were reported to the INSPECT system over the three-year study period. Numbers and types of controlled substances dispensed were similar to another study conducted during the years 2011, 2012, and 2013 in Indiana. Physicians, nurse practitioners, physician assistants, podiatrists, dentists, and pharmacists all queried the INSPECT system during the three-year study period.

Discussion/Conclusion: Ongoing analyses will further delineate trends in controlled substance dispensing and INSPECT use in Indiana. The effect of legislation and regulatory changes on controlled substance dispensing in Indiana will be determined.

Key words: controlled substances, prescription drug monitoring program, opioids, legislation, rescheduling, policy 


\section{Design of a Qualtrics Online Survey to Assess Prescription Drug Monitoring Program Use Using a Factorial Survey Design}

Brahmendra Reddy Viyyuri, PharmD

Graduate Student

University of lowa

College of Pharmacy

115 S Grand Ave

lowa City, IA 52242

brahmendrareddy-viyyuri@uiowa.edu

Matthew J Witry, PharmD, PhD

Assistant Professor

University of lowa

College of Pharmacy

115 S Grand Ave

lowa City, IA 52242

matthew-witry@uiowa.edu

Barbara St. Marie, PhD, ANP-BC, GNP-BC

Assistant Professor

University of lowa

College of Nursing

50 Newton Rd

lowa City, IA 52242

barbara-stmarie@uiowa.edu

\section{ABSTRACT}

Introduction: States have implemented prescription drug monitoring programs (PDMP) in an effort to manage opioid abuse epidemic. However, in many states, their use is voluntary. Little is known about the decision-making process of providers related to PDMP use.

Objectives: To assess the influence of patient and contextual related factors associated with the use of PDMP by healthcare providers.

Methods: The study uses a cross-sectional, interdisciplinary, factorial survey design to investigate the judgment process of providers. The study population include physicians, physician's assistants, nurse practitioners and pharmacists who care for patients in outpatient settings and have access to the lowa PDMP.

The survey was administered online using Qualtrics software. The survey design used vignette technique where respondents are presented with hypothetical patient prescriptions to evaluate and rate their likelihood of consulting the PDMP on a 0 -10 point scale. All vignettes consist of 9 dimensions (factors) related to patient, drug, prescriber and insurance but their values (levels) are systematically varied across the vignettes. The randomization process was fully automated using JavaScript in the Qualtrics. As such, we were able to generate a large number of random vignettes $(>50,000)$ with each respondent receiving a set of 8 vignettes. In addition to the vignettes, respondents are also asked to rank order the three primary concerns (drug diversion, patient misuse, safety), and to report their top five red flags that guide their decisions to consult the PDMP

Statistical Analysis: The unit of analysis is the vignette with the likelihood score (0-10 point) acting as the dependent variable. Predictor variables include vignette dimension values and provider characteristics. Multi-level models will be used for analysis to account for the clustering effects of responses given by the same respondent.

Keywords: prescription drug monitoring programs, PDMP, factorial survey, decision-making 


\section{Using the Theory of Planned Behavior to Explore Opioid Disposal Behaviors}

Arveen Kaur, PharmD., MPH

Graduate Student

University of Wisconsin

School of Pharmacy

777 Highland Ave

Madison, WI 53705, USA

akaur7@wisc.edu

David A Mott, PhD, FAPhA, RPh

Division Chair, Professor

University of Wisconsin

School of Pharmacy

777 Highland Ave

Madison, WI 53705, USA

David.mott@wisc.edu

\section{ABSTRACT}

Introduction: A high percentage of individuals are prescribed opioid medications every day. According to the CDC, prescription opioids (RXOpioids) are the leading cause of the opioid overdose epidemic in the United States. Sales of prescription opioids quadrupled between 1999 and 2014 and the average days' supply per opioid prescription increased from 13 days to 18 days between 2006 and 2015. Unused RXOpioids are a significant cause of opioid diversion and abuse. Commonly, unused RXOpioids are shared among friends or relatives, or stolen by a friend or relative. Proper disposal of RXOpioids could prevent diversion and abuse of unused RXOpioids. However, little research has assessed consumers' knowledge and attitudes about opioid disposal. There is a clear need for theoretically based research in this area to better understand consumer knowledge and behavior and to develop consumer-directed information to improve safe disposal behaviors.

Objective: The objectives of this study are to: (1) Assess patient's knowledge of safe disposal of RXOpioid medications (2) To assess patient's attitudes/belief, and motivation towards safe disposal of Rx Opioid medications.

Theoretical Framework: The Theory of Planned Behavior (TPB) serves as the theoretical model which will be used to elicit patients' knowledge, attitudes, and motivations regarding RXOpioid disposal behaviors. The TPB has been applied in many disciplines including pharmacy and public health. The TPB asserts that people's attitudes toward a behavior, their subjective norms, and perceived behavioral control influence people's behavioral intentions and ultimately their behaviors. Learning about patients' attitudes, perceived social norms, and behavioral control will provide information for developing targeted messages that will serve to increase patients' positive attitudes and perceptions about the behavior.

Proposed Method: Semi structured telephone interviews (constructed using Theory of Planned Behavior) will be conducted in Wisconsin in three different age groups of adults (18-25; 26-55; and 55+) recruited from different pharmacies. Theory of Planned Behavior will guide a total of 15 interviews-5 interviews in each age group. Transcripts of the semi-structured interviews will be qualitatively content analyzed, identifying themes related to major content areas.

Keywords: Disposal, Diversion, motivation, Rx Opioids 


\section{Establishing Inter-Reliability for a Coding Protocol for Analyzing Video Recordings for Opioid Consults Between Pharmacy Students and Standardized Patients}

Tanvee Thakur, B. Pharm, MS.

Graduate student

University of Wisconsin- Madison

College of Pharmacy

777 Highland Avenue,

Madison, WI- 53705

tmthakur@wisc.edu

Betty Chewning, PhD

Professor

University of Wisconsin- Madison

College of Pharmacy

777 Highland Avenue,

Madison, WI- 53705

Betty.chewning@wisc.edu

\section{ABSTRACT}

Introduction: It is necessary to inform patients who are prescribed opioids about the opioid specific dependency, potential for abuse and addictions and risks associated with opioids, particularly for an initial prescription. Pilot interviews with six pharmacists pointed out that pharmacists do not generally talk to patients about opioid specific risks during opioid medication consults. This study describes a coding protocol to extract reliable data from pharmacy student consults for opioid medications.

Objectives: To develop a reliable coding tool capable of identifying verbal and non-verbal communication patterns for opioid consults.

Methods: Consults were video recorded in a required communications class for third year student pharmacists in spring 2018 at the University of Wisconsin School of Pharmacy as part of a course quality improvement project. Pharmacy students were asked to consult patients as they would in natural settings. 71 consults were recorded, out of which 24 had limited English speaking patients. The coding protocol and codebook were developed to analyze verbal, non- verbal and limited English proficiency (LEP) consults. Two coders will be asked to code video recordings to evaluate inter- rater reliability. A process to establish a high value for Cohen's kappa will be established Cohen's kappa>0.85).

Results: This study is unique in analyzing video recordings, for focusing on opioid medications, for including LEP patients and for coding the non- verbal aspect of communication such as eye gaze. Reliability checks will be conducted intermittently during coding as well to maintain reliability throughout the coding process.

Discussion: Coding these consults reliably will help understand potential deficits in opioid consultations with patients. Importantly, it will help the course develop better training to train student pharmacists to communicate effectively with patients during opioid consults. This protocol can be easily adapted to a wide range of pharmacy student and pharmacist interventions to produce reliable data.

Key Words: Communication, opioids, pharmacy students, patients, dependency, abuse, inter- rater reliability, Cohen's kappa 